\title{
A Novel Haptic Feature Set for the Classification of Interactive Motor Behaviors in Collaborative Object Transfer
}

\author{
Zaid Al-Saadi ${ }^{1,2}$, Doganay Sirintuna ${ }^{1}$, Ayse Kucukyilmaz ${ }^{3}$, and Cagatay Basdogan ${ }^{1}$
}

\begin{abstract}
Haptics provides a natural and intuitive channel of communication during the interaction of two humans in complex physical tasks, such as joint object transportation. However, despite the utmost importance of touch in physical interactions, the use of haptics is under-represented when developing intelligent systems. This study explores the prominence of haptic data to extract information about underlying interaction patterns within physical human-human interaction (pHHI). We work on a joint object transportation scenario involving two human partners, and show that haptic features, based on force/torque information, suffice to identify human interactive behavior patterns. We categorize the interaction into four discrete behavior classes. These classes describe whether the partners work in harmony or face conflicts while jointly transporting an object through translational or rotational movements. In an experimental study, we collect data from 12 human dyads and verify the salience of haptic features by achieving a correct classification rate over 91\% using a Random Forest classifier.
\end{abstract}

Index Terms-Collaborative Manipulation, Classification, Dyadic Manipulation, Feature Extraction, Haptic Feedback, Machine Learning, Pattern Recognition, Performance Metrics, Physical Human-Human/Robot Interaction.

\section{INTRODUCTION}

$\mathbf{I}$ $\mathrm{N}$ recent years, a considerable amount of research has been directed toward designing robots to cooperate with humans in daily activities that require close physical interaction. One particular area of interest is collaborative object transportation, where human dyads would rely heavily on haptic sensations to coordinate their actions. Humans are sensitive to interactive behaviors during joint action, and can leverage haptic cues (i.e., the forces they apply and sense through their interactions with the manipulated object) to easily distinguish between interaction states, such as harmony and conflict. Such discrimination between interaction states is currently missing in physical human-robot interaction (pHRI), and collaboration can be improved by making the robot proactive against changes in interaction behaviors.

We trust that understanding physical human-human interactions (pHHI) is crucial to implement a robotic system that is able to classify dyadic interactive behaviors. This study is an

${ }^{1}$ Z.A., D.S., and C.B. are with the College of Engineering, Koç University, Istanbul, 34450, Turkey. E-mail: \{zalsaadi13, dsirintuna13, cbasdogan\}@ku.edu.tr.

${ }^{2}$ Z.A. is with the Al-Khwarizmi College of Engineering, University of Baghdad, Baghdad, 10070, Iraq.

${ }^{3}$ A.K. is with the School of Computer Science, University of Nottingham, Nottingham, NG8 1BB, UK. E-mail: Ayse.Kucukyilmaz@nottingham.ac.uk.

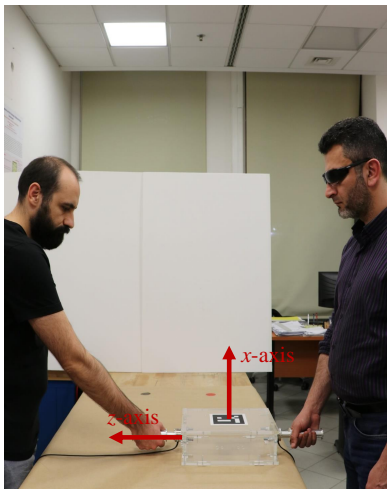

(a)

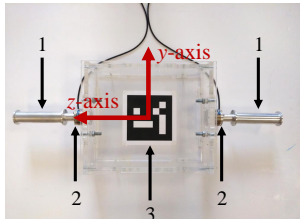

(b)

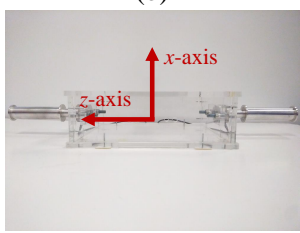

(c)
Fig. 1: (a) Two humans cooperate to manipulate an object from one location to another. (b) and (c) are the top and the front views of the manipulated object, respectively. The object coordinate frame is shown in red. The numbers 1,2 , and 3 in (b) highlight the handles, the F/T sensors, and the ArUco marker, respectively.

effort to provide insight into the inherently important role of haptics in differentiating distinct interaction behavior classes during the collaboration of two physically-linked partners. The findings of our study will work as a basis for future work to enable proactive robotic partners that can accommodate varying interactive behaviors. To the best of our knowledge, ours is the first study to design interaction-specific haptic features to improve identification of conflict-related pHHI behaviors in physical co-manipulation.

In this paper, we report evidence that harmonious and conflicting behavior patterns in pHHI can be recognized using haptic information alone. In doing so, we present an experimental study with 12 subjects in a 6-DoF object manipulation task, as shown in Figure 1 (Section III). Six task scenarios are designed, in each of which at least two of four specific interaction behavior patterns are generated: 1) Harmonious Translation (HT), 2) Conflicting Translation (CT), 3) Harmonious Rotation (HR), and 4) Conflicting Rotation (CR).

In order to distinguish between these interaction classes, we define features based on the metrics devised by Noohi and Žefran to quantify the collaboration quality during a dyadic manipulation task [1]. As these metrics are originally defined for a vertical translation task, they do not capture torquerelated information, which is essential to describe rotational motion in a 6-DoF task. To address this, we combine force and torque information and design wrench-based metrics as described in Section IV.

The feature extraction and classification methodology is 
presented in Section V. For the classification of interaction behaviors, we train a Random Forest classifier [2]. Our results indicate that the trained classifier achieves a correct classification rate over $91 \%$, underlining the prominent influence of haptics in distinguishing between interactive behavior classes. These results are discussed in detail in Section VI, followed by conclusions in Section VII.

\section{BACKGROUND}

Recent literature indicates that haptic feedback plays an important role in improving the task performance of interacting partners in virtual environments [3]-[10], when working with real robots [11], [12], and in pHHI [13].

In addition to performance benefits, haptics has a communicative function. Takagi et al. [9] explored the effect of haptic communication on dyads' performance in a dynamic target tracking task. They suggested that the coupling dynamics may determine the amount of information that can be communicated among physically interacting partners. Mielke et al. [13] showed that when two humans collaboratively move an object, lateral movements were triggered by a specific force profile applied by the leader. Using this force profile, the intention for future motion could be predicted. Later, the same group showed that linear and angular velocity of the co-manipulated object can be used to accurately estimate the human motion intention (i.e., translation vs. rotation) [14]. Parastegari et al. [12] designed a controller for robot-to-human handover, where they used the pulling force applied on the object, the object acceleration, and the relative motion between the object and the robot hand, to achieve smooth and safe handover. Mörtl et al. [15] proposed effort sharing policies through a dynamic role allocation, based on a force-based agreement criteria, and showed that dynamic autonomy improves task completion time and energy requirements of the human. To understand such negotiation behaviors better, Oguz et al. [16] programmed robotic behaviors based on game theory and illustrated that haptics enables humans to understand these behaviors more accurately.

In human studies, haptics has been used to assess the performance of dyad in manipulation tasks. Noohi and Žefran [1] investigated the quality of collaboration in physical humanhuman interaction while two partners lift an object vertically. They proposed a set of quantitative metrics that evaluate the performance of haptically-coupled subjects, and crossvalidated those metrics with the subjects' self-assessments. These metrics were valid for a 1D translation task, and assessed the performance of the partners in terms of the efficiencies of individuals, the team, and their negotiation, as well as the similarity of forces. In this study, we designed our features through inspiration from this study. We modified these metrics to support a 6-DoF task by reformulating the metrics to handle torques as well as forces in the 3D Cartesian space.

Another interesting observation arising from human studies is about role allocations between partners during dyadic interaction [6], [17]. Reed and Peshkin [18] observed that when two human partners perform a dyadic target acquisition task, two opposing roles, responsible for accelerating or decelerating the task, were adopted by collaborating humans. Stefanov et al. [19] also observed two roles in human-human haptic interaction, namely the executor and the conductor of the task. Both studies identified the roles regarding interaction forces and velocities. On the other hand, Takagi et al. [20] examined rigidly coupled dyadic reaching movements. Although their study showed that the dyads did not coordinate during joint reaching movements towards to the same target, the collaborating partners still displayed "accelerating" versus "decelerating" roles. However, they explained these acceleration/deceleration roles by the speed mismatch between the partners and the differences in their movement reaction times.

Even though the literature is rich in terms of defining human intentions and partner roles in dyadic interaction, there are only a few studies that focus on collaborative tasks as an attempt to define possible interaction patterns. Melendez-Calderon et al. [21] defined five human interaction patterns for a tracking task. This study provided static and task-dependent roles. A rule-based quantitative classification technique, depending on the interaction torques and EMG recordings from partners, was proposed to identify these roles and discuss their efficiencies for specific tasks. Jarrassé et al. [22] presented a comprehensive taxonomy of interactive behaviors, namely competition, collaboration, and cooperation. They proposed a utility-based game theoretic approach to describe and implement interactive behaviors, where cost functions are defined to distinguish between interaction classes. This approach was later demonstrated on a simple reaching task with a robot [23]. Agravante et al. [24] introduced a taxonomy for collaborative object transportation by classifying the grasp types and the relative pose between the agents. They decomposed a complex transportation task into subtasks, and used a finite state machine to program a robot to perform the task collaboratively with a human partner. In our earlier work [25], we proposed a hierarchical taxonomy for $\mathrm{pHHI}$, defining three main classes of collaborative interaction types, namely harmonious, conflicting and neutral behaviors. In contrast to aforementioned studies, our taxonomy in [25] looks at general interaction states and provides a simpler characterization for physical collaboration; hence, [25] will be used in this study to define the behavior classes.

Our hypothesis is that haptic-related features would be sufficient to distinguish between interactive behavior patterns without the need to include the kinematic information, eliminating the need to track the manipulated object. In [25], we investigated the use of raw interaction data, and extracted features by processing the partners' applied forces and the manipulated object's velocity from annotated data. Later, we proposed an online feature extraction method and demonstrated its use for identifying interaction patterns in real-time collaboration using similar features [26]. In both studies, we observed that raw haptic data were insufficient to achieve good classification rates and the object pose information was needed. Hence, in this study, we utilize richer haptic features derived from raw data to improve the accuracy in classifying interaction behaviors without a need for object pose information. Selected features isolate haptics-related information and aim to demonstrate the prominence and sufficiency of haptics 
in explaining interaction states during pHHI.

\section{EXPERIMENT}

An experiment is designed to create a dataset of pHHI behaviors. The experimental task requires two human partners to collaborate in order to lift, move, and place an object to a target configuration through translational and rotational movements. This section describes the experimental setup and the protocol, and details the data collection and preparation process.

\section{A. Experimental Apparatus and Data Collection}

To enable data collection, we manufactured a box-shaped object, which can be easily co-manipulated by two subjects (see Figure 1). The object weighs $3100 \mathrm{~g}$ in total. Its body is made of plexiglass with dimensions of $30 \mathrm{~cm} \times 25 \mathrm{~cm} \times 10 \mathrm{~cm}$ and weighs $2538 \mathrm{~g}$. Two aluminum handles, each weighing $231 \mathrm{~g}$, were attached to facing sides of the object. Two ATIMini40 force/torque (F/T) sensors, each weighing $50 \mathrm{~g}$, were placed between the handles and the object body to record the forces and torques applied on the side of each subject. These signals were sampled by a USB X Series NI data acquisition system at $1 \mathrm{kHz}$ frequency.

The torques applied by each agent on the object are computed as the cross product of the force measured by the $\mathrm{F} / \mathrm{T}$ sensor and the position vector defining the point of application (i.e., the sensor) with respect to the object's centre of mass. The computed torque is a vector quantity: $\boldsymbol{\tau}^{c}=\left[\begin{array}{lll}\tau_{x}^{c}, & \tau_{y}^{c}, \tau_{z}^{c}\end{array}\right]^{\top}$. It's important to note that due to the placement of the sensors with respect to the users' interaction points (see Figure 1), some torques are generated at the handles during a manipulation, which are measured directly by the sensors $\boldsymbol{\tau}^{m}=\left[\tau_{x}^{m}, \tau_{y}^{m}, \tau_{z}^{m}\right]^{\top}$. As a result, we calculate the total torque exerted on each partner's side by summing up these two torque components as

$$
\boldsymbol{\tau}=\left[\tau_{x}^{c}+\tau_{x}^{m}, \tau_{y}^{c}+\tau_{y}^{m}, \tau_{z}^{c}+\tau_{z}^{m}\right]^{\top}
$$

The object's position were tracked by Basler acA 192025um USB 3.0 camera using an ArUco marker [27] and the OpenCV library. The marker was placed on the top of the object as shown in Figure 1.

\section{B. Interaction Patterns}

Following Madan et al.'s taxonomy of interactive behaviors [25], we defined four interaction patterns to be examined in this study. Two of these are harmonious interaction types with a common intention to start/continue the motion, whereas the remaining two are conflicting interaction types:

(1) Harmonious Translation (HT): The partners agree on translating the object towards a common target configuration. (2) Conflicting Translation (CT): The dyad faces a conflict as only one of the partners intends to translate the object to the target configuration.

(3) Harmonious Rotation (HR): The partners share the same rotation intention, hence they rotate the object collaboratively.

(4) Conflicting Rotation (CR): The dyad faces a conflict as only one of the partners intends to rotate the object.

\section{Experimental Scenarios}

During the experiment, the dyad was instructed to move the object collaboratively in mid-air to visit a set of target configurations, reachable through primitive translation and rotation movements. We artificially created harmonious and conflicting behaviors by providing one of the partners with different target configurations. The role of this partner was always played by the experimenter. This was deliberately done to eliminate implicit role allocations and differences in how people respond to and resolve conflicts. The subjects were briefed that the experimenter will be the supervisor of the task and they should aid him to complete the task in case they felt conflicts.

In return, the subjects' task sequence was always constant and asked them to: (1) lift the object up $(U),(2)$ rotate it counterclockwise about the $x$-axis $\left(R_{x}^{+}\right)$, (3) translate along the negative $z$-axis into the red target configuration marked on the table $\left(T_{z}^{-}\right)$, (4) translate along the $y$-axis to reach the black target configuration $\left(T_{y}^{+}\right)$, (5) rotate the object clockwise about $x$-axis $\left(R_{x}^{-}\right)$, and (6) put it down $(D)$. In shorter form, the subject's task sequence can be written as $\left\{U, R_{x}^{+}, T_{z}^{-}, T_{y}^{+}, R_{x}^{-}, D\right\}$. Note that, in this paper, all the movement axes refer to the local object coordinates unless stated differently.

The experimenter, on the other hand, was given different target configurations, defining six different scenarios, which are depicted in Figure 2(a). Each of these six scenarios aims at isolating more than one of the four specific interaction patterns. The primitive motions that shall be performed by the subject and the experimenter in each scenario are summarised in Table I. The table also indicates the anticipated interaction patterns arising from the interplay between the intended motions of the experimenter and the subject. Figure 2 shows the six scenarios ( $\mathrm{Sc} 1-\mathrm{Sc} 6)$ followed by the experimenter and a representative experimenter-subject scenario $(\mathrm{Sc} 3)$ with the expected behaviors.

\section{Participants and Protocol}

We conducted a study with 12 subjects ( 8 males and 4 females with an average age of $28.4 \mp 7.0 \mathrm{SD}$ ) to collect data within a dyadic object transportation task. During the experiment, each subject collaborated with the experimenter to move the object between designated configurations following the patterns in Table I. The experimenter always stood at the same position and acted as the task supervisor. In order to eliminate any visual contact with the experimenter, the subjects wore industrial eye protection glasses which were modified by shading most of the glasses, leaving a small gap at the bottom to observe just the scene.

The subjects started the experiment facing the experimenter, while the object lay on the table. The subjects and the experimenter were instructed to grasp the handle on their side, lift the object up from the table, and move and place it to a target pose by passing through a series of intermediate goal configurations. The dyad performed 12 trials, where scenarios Sc1-Sc6 were executed twice (Table I). The order of the 


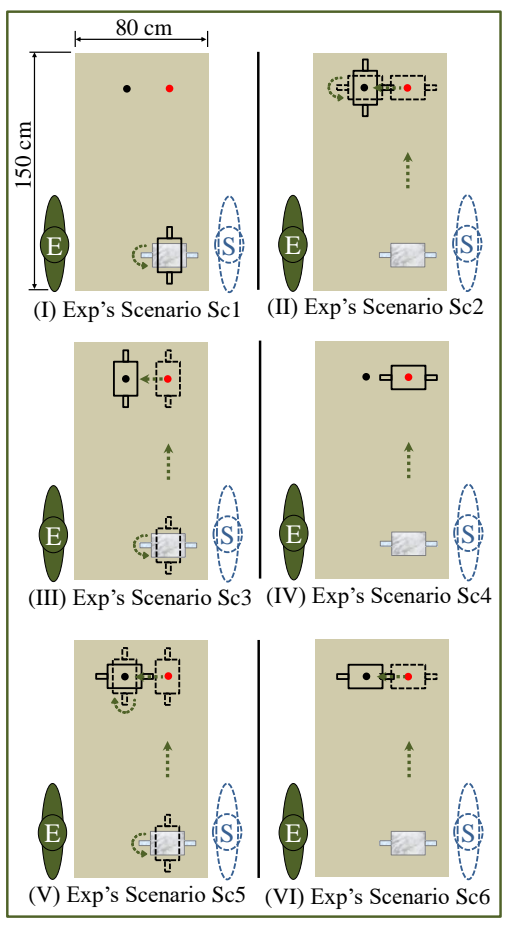

(a) Experimenter's Scenarios

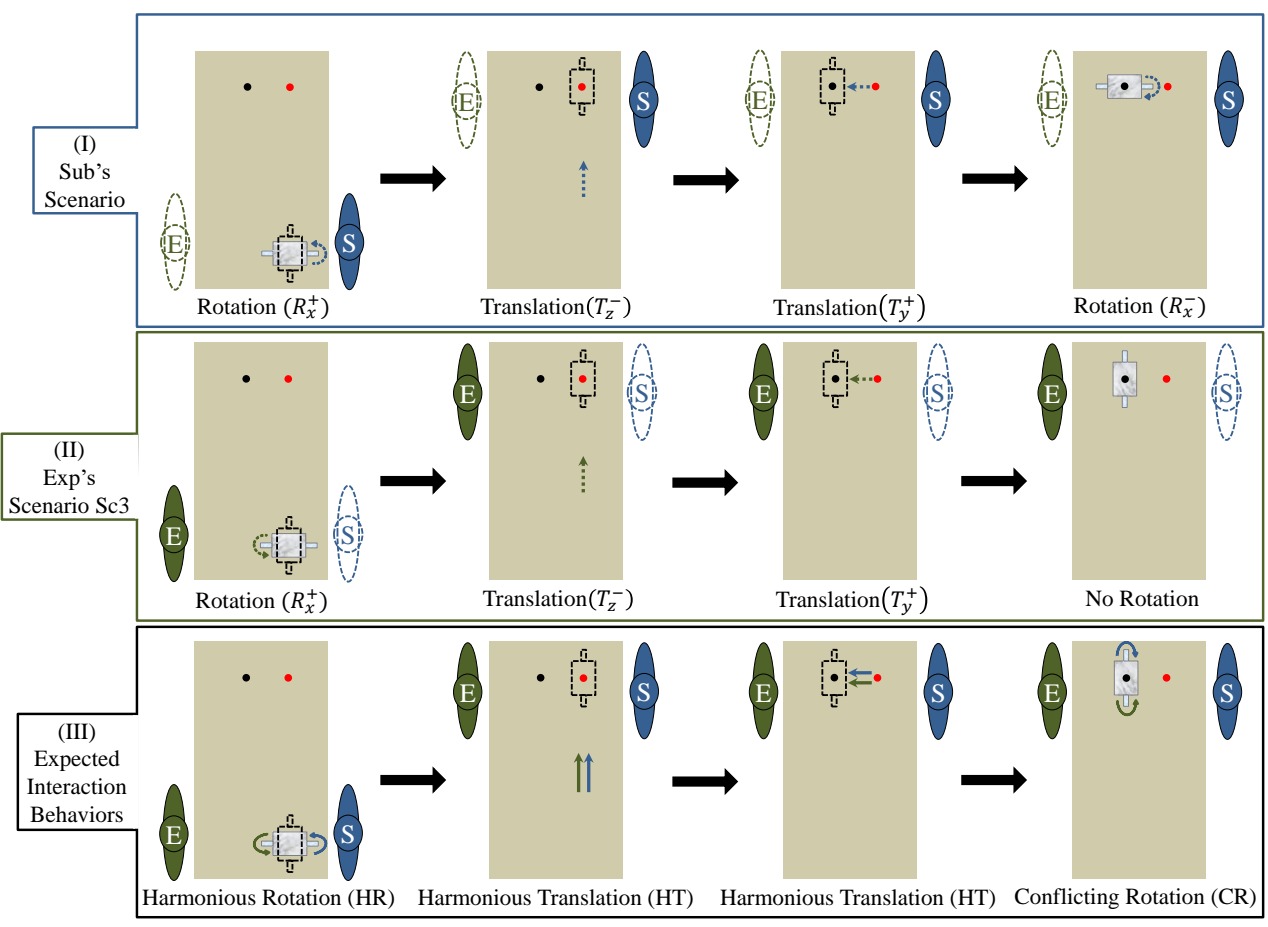

(b) Experimenter-Subject Representative Scenario

Fig. 2: (a.I-a.VI) Experimenter's (E: Exp) scenarios Sc1-Sc6, (b.I) Subject's (S: Sub) scenario, consisting of the task sequence $\left\{U, R_{x}^{+}, T_{z}^{-}, T_{y}^{+}, R_{x}^{-}, D\right\}$, where $U$ : lift the object Up, $D$ : put the object Down, $R$ : Rotation, $T$ : Translation. Superscripts and subscripts for $R$ and $T$ refer to the direction and axis of the transformation, respectively. For simplicity $U$ and $D$ are omitted from the figure, (b.II) Individual steps of actions in Experimenter's scenario Sc3: $\left\{U, R_{x}^{+}, T_{z}^{-}, T_{y}^{+}, D\right\}$, (b.III) Expected interaction patterns generated due to the

interplay between the Subject's and the Experimenter's actions. The red and black dots represent the intermediate and final object configurations as shown to the subject. Dashed arrows represent the partners' intentions, whereas solid arrows represent their actions.

TABLE I

PRIMITIVE MOTIONS WITHIN THE EXPERIMENTAL SCENARIOS AND THE ANTICIPATED INTERACTION BEHAVIORS. S: SUbJECT, E: EXPERIMENTER, SC1-SC6: EXPERIMENTER'S SCENARIOS. $U$ : LIFT THE OBJECT UP, $D$ : PUT THE OBJECT DOWN, $R$ : ROTATION, $T$ : TRANSLATION. THE SUPERSCRIPTS AND SUBSCRIPTS FOR $R$ AND $T$ REFER TO THE DIRECTION AND AXIS OF THE TRANSFORMATION, RESPECTIVELY.

\begin{tabular}{|l|c|c|c|c|c|c|c|}
\hline & \multicolumn{6}{|c|}{ Primitive Motion (PM) and Expected Behavior (EB) } \\
\hline \hline S & PM & $U$ & $R_{x}^{+}$ & $T_{z}^{-}$ & $T_{y}^{+}$ & $R_{x}^{-}$ & $D$ \\
\hline \hline \multirow{2}{*}{ E: Sc1 } & PM & $U$ & $R_{x}^{+}$ & - & $D$ & - & - \\
& EB & & $\mathrm{HR}$ & $\mathrm{CT}$ & - & - & - \\
\hline \multirow{2}{*}{ E: Sc2 } & PM & $U$ & - & $T_{y}^{+}$ & $T_{z}^{+}$ & $R_{x}^{+}$ & $D$ \\
& EB & & $\mathrm{CR}$ & $\mathrm{HT}$ & $\mathrm{HT}$ & $\mathrm{HR}$ & - \\
\hline \multirow{2}{*}{ E: Sc3 } & PM & $U$ & $R_{x}^{+}$ & $T_{z}^{-}$ & $T_{y}^{+}$ & - & $D$ \\
& EB & & $\mathrm{HR}$ & $\mathrm{HT}$ & $\mathrm{HT}$ & $\mathrm{CR}$ & - \\
\hline \multirow{2}{*}{ E: Sc4 } & PM & $U$ & - & $T_{y}^{+}$ & - & $D$ & - \\
& EB & & $\mathrm{CR}$ & $\mathrm{HT}$ & $\mathrm{CT}$ & - & - \\
\hline \multirow{2}{*}{ E: Sc5 } & PM & $U$ & $R_{x}^{+}$ & $T_{z}^{-}$ & $T_{y}^{+}$ & $R_{x}^{-}$ & $D$ \\
& EB & & $\mathrm{HR}$ & $\mathrm{HT}$ & $\mathrm{HT}$ & $\mathrm{HR}$ & - \\
\hline \multirow{2}{*}{ E: Sc6 } & PM & $U$ & - & $T_{y}^{+}$ & $T_{z}^{+}$ & - & $D$ \\
& EB & & $\mathrm{CR}$ & $\mathrm{HT}$ & $\mathrm{HT}$ & $\mathrm{CR}$ & - \\
\hline
\end{tabular}

scenarios was randomised, but was the same throughout the experiment for all subjects.

In order to achieve synchronization, we implemented two distinctive software beeps: The first beep indicated the partners to grasp the handles gently. The second beep was played after five seconds to indicate the start of the trial, asking the dyad to lift the object up and start the transporting motion.

At the beginning of the experiment, the subjects were briefed about the task, and familiarized themselves with the object. The subjects were given training through experimenting with the task sequence prior to data acquisition. During the training, each subject performed all the scenarios with the experimenter (Sc1-Sc6) once. The subjects were not aware of the experimenter's goal, but they were told that in some trials, the experimenter might have different goal configurations at each step within the task. In such cases, the subjects were asked to collaborate with the experimenter. For instance, if they felt a conflict when trying to rotate the object, they were instructed to stop the rotation and move on to the next task segment along with the experimenter.

\section{E. Data Segmentation}

After data collection, raw haptic data were annotated by the experimenter, who has a good understanding of the interaction behaviors. The annotation was done manually through visual inspection of data based on the expected patterns listed in Table I. Although the primitive motions are designed to elicit anticipated interaction patterns, we further confirmed that these interaction patterns appear in the data. The time-series was first segmented by utilizing the object pose. However, as the pose information was expected to be static during conflicting behaviors, we used the changes in force and torque commands to segment CT and CR. For instance, the conflicting translation 
periods were segmented using the changes in forces applied in the direction of conflict. Likewise, utilizing the changes in the applied torques, the conflicting rotation periods were tightly segmented. The remaining periods, where no changes are observed in the applied forces/torques, were considered as pauses. After this procedure, each segment was assigned a label, matching one of the four interaction behavior patterns. The haptic data was recorded at a frequency of $1 \mathrm{kHz}$, and for each time step of our labeled data, the haptic-based features were calculated. Detailed information on the labelling and segmentation processes can be found in Appendix A.

\section{HAPTIC-RELATED MEtrics FOR DyAdiC COLLABORATION}

Our hypothesis in this study is that haptic information plays an essential role for the classification of interaction patterns. In our earlier work, we showed that the raw haptic data were insufficient to achieve good classification rates and the kinematic information of manipulated object was needed [25] [26]. However, this requires object tracking, which can typically be achieved through external sensing. In this study, we eliminate the need for object pose by designing a set of haptics-related features to train the behavior classification model. These features are computed based on the metrics proposed by Noohi and Žefran to quantify the collaboration quality on a 1-DoF task [1]. In this section, we present these metrics and provide details on how we modified them to handle 6-DoF motion.

In order to put the following equations in context, assume the scenario, where the experimenter $(E)$ and the subject $(S)$ exert forces $\boldsymbol{f}_{E}$ and $\boldsymbol{f}_{S}$ on an object. The resultant net force acting on the object is then computed as $\boldsymbol{f}_{\text {sum }}=\boldsymbol{f}_{E}+\boldsymbol{f}_{S}$. We decompose the force applied by each partner into two components: an effective force component that is in the direction of $f_{\text {sum }}$ and contributes to the task, and a normal component, that is canceled out during the interaction by the forces applied by the other partner in the normal direction. The effective forces $\boldsymbol{f}_{E}^{*}$ and $\boldsymbol{f}_{S}^{*}$, applied on the object by the experimenter and the subject, are computed respectively as the projection of $\boldsymbol{f}_{E}$ and $\boldsymbol{f}_{S}$ on the net force, $\boldsymbol{f}_{\text {sum }}$, as shown in Figure 3. Since the effective forces contribute to the net force in variable degrees, they can be written as:

$$
\begin{aligned}
& \boldsymbol{f}_{E}^{*}(t)=\alpha \boldsymbol{f}_{\text {sum }}(t) \\
& \boldsymbol{f}_{S}^{*}(t)=\beta \boldsymbol{f}_{\text {sum }}(t),
\end{aligned}
$$

where $\alpha+\beta=1$, and $t$ stands for the current time step.

The force cooperation index $\delta$ represents the extent to which the partners help each other and can be computed as:

$$
\delta=1 / 2-\alpha,
$$

where $\alpha$ is defined in (2). $\delta=0$ indicates that each partner carries out half of the voluntary effort during the task. When $|\delta(t)| \leq 0.5$, partners work in a collaborative mode: either both partners exert forces in the direction of the net force as seen in Figure 3(b), or one partner is passive as seen in Figure 3(a). In contrast, when one partner's force is in the opposite direction to the net force, as in Figure 3(c), the partners work in a non-collaborative mode with $|\delta(t)|>0.5$.

In the rest of this section, we summarize the force-related metrics, which are used as features for behavior classification.

Individual efficiency: This assesses to what extent a partner's exerted force contributes to his/her effective force:

$$
M_{e_{k}}^{f}(t)=\frac{\left\|\boldsymbol{f}_{k}^{*}(t)\right\|}{\left\|\boldsymbol{f}_{k}(t)\right\|}
$$

where $k$ refers to the experimenter (E) or the subject $(\mathrm{S})$ and $0 \leq M_{e_{k}}^{f}(t) \leq 1$. The individual efficiency is maximized when $M_{e_{k}}(t)=1$.

Team efficiency: The efficiency of the dyad as a team is defined as the ratio of the magnitude of the net force to the sum of the magnitudes of individual forces exerted by partners:

$$
M_{t e}^{f}(t)=\frac{\left\|\boldsymbol{f}_{\text {sum }}(t)\right\|}{\left\|\boldsymbol{f}_{E}(t)\right\|+\left\|\boldsymbol{f}_{S}(t)\right\|}
$$

where $0 \leq M_{t e}^{f}(t) \leq 1$. When $M_{t e}^{f}(t)=1$, no effort is wasted, i.e., the individual forces and the effective forces are the same and $\left\|\boldsymbol{f}_{\text {normal }}(t)\right\|=0$; hence team efficiency is maximum. When $M_{t e}^{f}(t)=0$, the partners cancel each other' forces, i.e., $\left\|\boldsymbol{f}_{\text {sum }}(t)\right\|=0$; hence team efficiency is minimum.

Negotiation efficiency: The efficiency of the negotiation process represents the extent of disagreement between the partners at a given time during the task:

$$
M_{n e}^{f}(t)=\frac{\left\|\boldsymbol{f}_{\text {sum }}(t)\right\|}{\left\|\boldsymbol{f}_{E}^{*}(t)\right\|+\left\|\boldsymbol{f}_{S}^{*}(t)\right\|}
$$

where $0 \leq M_{n e}^{f}(t) \leq 1$. Note that whenever the interaction is in a collaborative mode, $|\delta(t)| \leq 0.5$, the negotiation efficiency is maximum, such that $M_{n e}^{f}(t)=1$. Otherwise, $M_{n e}^{f}(t)<1$.

Similarity of forces: This represents the similarity between the effective forces exerted individually by partners:

$$
M_{s}^{f}(t)=1-\left|\frac{\left\|\boldsymbol{f}_{E}^{*}(t)\right\|-\left\|\boldsymbol{f}_{S}^{*}(t)\right\|}{\left\|\boldsymbol{f}_{\text {sum }}(t)\right\|}\right|
$$

where $0 \leq M_{s}^{f}(t) \leq 1$. When the partners work in collaborative mode $(|\delta(t)| \leq 0.5)$, we have $0<M_{s}^{f}(t) \leq 1$. Maximum similarity, $M_{s}^{f}(t)=1$, means that both partners exert exactly half of the voluntary effort. When $M_{s}^{f}(t)=0$, the partners are in non-collaborative mode $(|\delta(t)|>0.5)$.

In Appendix A, we present some data captured during the experiment, to present observations on patterns encountered in each behavior. The data indicate that some interaction

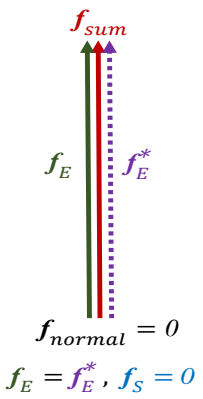

(a) One active partne

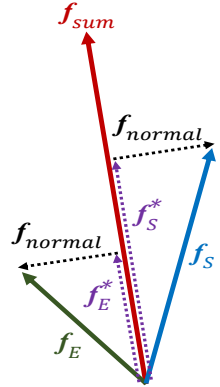

(b) Cooperative

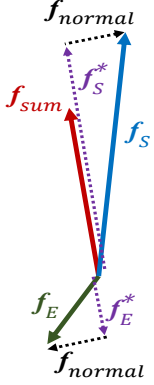

(c) Non-cooperative
Fig. 3: Force decomposition (adopted from Noohi and Žefran [1]) 
behaviors exhibit very similar force patterns, but different torque patterns. As a result, the force-related metrics will be insufficient to describe interaction behaviors. Therefore, we extend the above formulations to define torque-related metrics as an effort to extract more comprehensive and informative features. Table II provides the relevant formulations for all the metrics, consisting of both force and torque-based information. These metrics are called "wrench-based" in the rest of this paper.

TABLE II

WRENCH-BASED METRICS.

\begin{tabular}{|ll|}
\hline Metric & Formula \\
\hline Individual force efficiencies & $M_{e_{k}}^{f}(t)=\frac{\left\|\boldsymbol{f}_{k}^{*}(t)\right\|}{\left\|\boldsymbol{f}_{k}(t)\right\|}, k \in E, S$ \\
\hline Team force efficiency & $M_{t e}^{f}(t)=\frac{\left\|\boldsymbol{f}_{\text {sum }}(t)\right\|}{\left\|\boldsymbol{f}_{E}(t)\right\|+\left\|\boldsymbol{f}_{S}(t)\right\|}$ \\
\hline Negotiation force efficiency & $M_{n e}^{f}(t)=\frac{\left\|\boldsymbol{f}_{\text {sum }}(t)\right\|}{\left\|\boldsymbol{f}_{E}^{*}(t)\right\|+\left\|\boldsymbol{f}_{S}^{*}(t)\right\|}$ \\
\hline Similarity of forces & $M_{s}^{f}(t)=1-\left|\frac{\left\|\boldsymbol{f}_{E}^{*}(t)\right\|-\left\|\boldsymbol{f}_{S}^{*}(t)\right\|}{\left\|\boldsymbol{f}_{\text {sum }}(t)\right\|}\right|$ \\
\hline Individual torque efficiencies & $M_{e_{k}}^{\tau}(t)=\frac{\left\|\boldsymbol{\tau}_{k}^{*}(t)\right\|}{\left\|\boldsymbol{\tau}_{k}(t)\right\|}$ \\
\hline Team torque efficiency & $M_{t e}^{\tau}(t)=\frac{\left\|\boldsymbol{\tau}_{\text {sum }}(t)\right\|}{\left\|\boldsymbol{\tau}_{E}(t)\right\|+\left\|\boldsymbol{\tau}_{S}(t)\right\|}$ \\
\hline Negotiation torque efficiency & $M_{n e}^{\tau}(t)=\frac{\left\|\boldsymbol{\tau}_{\text {sum }}(t)\right\|}{\left\|\boldsymbol{\tau}_{E}^{*}(t)\right\|+\left\|\boldsymbol{\tau}_{S}^{*}(t)\right\|}$ \\
\hline Similarity of torques & $M_{s}^{\tau}(t)=1-\left|\frac{\left\|\boldsymbol{\tau}_{E}^{*}(t)\right\|-\left\|\boldsymbol{\tau}_{S}^{*}(t)\right\|}{\left\|\boldsymbol{\tau}_{s u m}(t)\right\|}\right|$ \\
\hline
\end{tabular}

\section{Feature Extraction and Classifier Design}

At the end of the experiment, the raw data were processed to create a labelled dataset of wrench-based metrics as features. Raw time-series was segmented and labelled based on the segmentation method explained in Section III-E.

\section{A. Feature Extraction}

Although the object movements are mainly in the $y$ - $z$ plane for the current task, we are interested in classification for unconstrained transportation tasks. As a result, we chose not to limit our feature extraction approach to only the $y-z$ plane. However, the nature of our task implies load bearing, hence we record comparably large forces in $x$-axis and torques around $y$ axis due to the lifting motion. Although not directly pertinent to the interaction patterns, these could dominate subtle force and torque patterns which could be relevant to the interaction dynamics. Hence, in addition to extracting features within the 6-DoF task space, we separately analysed the task within three principal orthogonal movement planes in the Cartesian space, i.e., $x-y, x-z$, and $y-z$ as follows:

Let's assume $A$ refers to a specific plane $A \in\{x-y, x-z, y$ $z\}$. The forces exerted on $A$ at the experimenter's side, $\boldsymbol{f}_{E_{A}}$, and the subject's side, $\boldsymbol{f}_{S_{A}}$, were computed using a projection operation. The net force applied on the object by the partners on plane A was computed as $\boldsymbol{f}_{\text {sum }_{A}}=\boldsymbol{f}_{E_{A}}+\boldsymbol{f}_{S_{A}}$. The effective forces applied by the dyad, $\boldsymbol{f}_{E_{A}}^{*}$ and $\boldsymbol{f}_{S_{A}}^{*}$, were computed as the projection of $\boldsymbol{f}_{E_{A}}$ and $\boldsymbol{f}_{S_{A}}$ on $\boldsymbol{f}_{\text {sum }_{A}}$ as explained in Section IV. In order to perform torque decomposition, we considered the torques around the axis perpendicular to plane $A$ for each partner $\left(\tau_{E_{A}}\right.$ and $\tau_{S_{A}}$ for the experimenter and the subject, respectively). Accordingly, the net torque applied by the partners on the object around the axis perpendicular to the plane $A$ was computed as $\tau_{\text {sum }_{A}}=\tau_{E_{A}}+\tau_{S_{A}}$. Because $\tau_{E_{A}}$ and $\tau_{S_{A}}$ are in the same direction of $\tau_{\text {sum }}$, they also stand as the effective torques (i.e., $\tau_{E_{A}}^{*}=\tau_{E_{A}}$ and $\tau_{S_{A}}^{*}=\tau_{S_{A}}$ ).

After performing force and torque decomposition separately on each principal movement plane $(x-y, x-z$, and $y-z)$, we computed the wrench-based features listed in Table III.

TABLE III

WRENCH-BASED FEATURES

\begin{tabular}{|c|c|c|c|}
\hline \multirow{2}{*}{ Features } & \multirow{2}{*}{ Description } & \multicolumn{2}{|c|}{ No. of Features } \\
\hline & & Space-Based & Plane-Based \\
\hline $\begin{array}{c}M_{e_{E}}^{f}, M_{e_{E}}^{\tau}, \\
M_{e_{S}}^{f}, M_{e_{S}}^{\tau}\end{array}$ & Individual efficiencies & $1 \times 4$ & $3 \times 4$ \\
\hline$M_{t e}^{f}, M_{t e}^{\tau}$ & Team efficiency & $1 \times 2$ & $3 \times 2$ \\
\hline$M_{n e}^{f}, M_{n e}^{\tau}$ & Negotiation efficiency & $1 \times 2$ & $3 \times 2$ \\
\hline$M_{s}^{f}, M_{s}^{\tau}$ & Similarity & $1 \times 2$ & $3 \times 2$ \\
\hline & Total & 10 & 30 \\
\hline
\end{tabular}

\section{B. Classifier Design}

We used a random forest classifier to distinguish between the aforementioned interaction patterns. Random forest is an ensemble learning algorithm, which combines multiple decision trees, i.e., a forest. This classifier was chosen as it does not perform any feature transformation (i.e., the features can be ranked for individual usefulness) and training and testing the model is faster than competitive algorithms, such as SVMs, or deep learning based methods. The algorithm works by learning decision trees on different subsamples from the dataset and uses averaging to select the correct class for multiclass classification given a multitude of decision tree results. In this study, we used the Python Scikit-Learn implementation of the algorithm [28]. The number of trees in the random forest was set to 100 , the maximum depth of the trees were unconstrained (i.e., the nodes were expanded until all leaves are single samples), and the quality of a split is measured through information gain.

In our experiments, we investigated the use of different feature sets. To evaluate which feature set is superior to others, we trained a separate classifier for each feature type. To verify the classification performance and to avoid overfitting, each dataset (consisting of the features and corresponding behavior labels) was split into two distinct parts as the training and test sets. The splitting was done by selecting data coming from 8 trials from all the subjects as the training set, and the remaining data of 4 trials as the test set. As a result, the training set was used to learn the classification model, whereas the test set was reserved for assessing the performance of the trained classifier. The classifier was evaluated using the correct classification rate (accuracy), normalised confusion matrix, and the balanced error rate (BER).

Once we assessed the overall success of each feature set, we selected the top achiever, and investigated how the corre- 


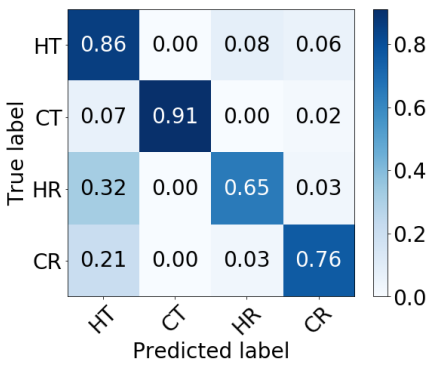

(a)

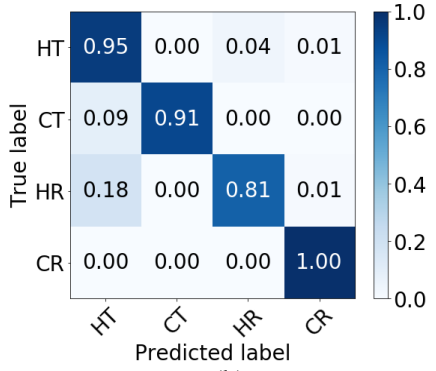

(b)
Fig. 4: Confusion matrices for a) classifier trained with WSB feature set and b) classifier trained with WPB feature set. HT, CT, $\mathrm{HR}$, and $\mathrm{CR}$ denote harmonious translation, conflicting translation, harmonious rotation, and conflicting rotation, respectively.

sponding classifier performs when the test data comes from a subject, who was not included in the training set. For this purpose, we conducted leave-one-out (L1O) cross validation, where we used the data from 11 subjects as the training set, and assessed the performance of the trained classifier using the $12^{\text {th }}$ subject's data as the test set. The procedure was repeated looping through all subjects, so that each subject's data was isolated once over the analysis. In addition, we performed feature selection on the top achiever to analyse which subset of features optimize the classification accuracy.

\section{RESULTS AND DISCUSSION}

This section summarises the classification results with the proposed feature set, followed by a discussion of a subsequent feature selection analysis to develop an optimal feature set, which is most informative for the task at hand.

\section{A. Classification with Wrench-based Feature Set}

We have extracted features through two decomposition approaches explained in Section V:

WSB Wrench Space-Based Feature Set: Features are extracted using the 10 force/torque-related metrics computed using space-based decomposition approach (i.e., 5 forcerelated metrics and 5 torque-related metrics).

WPB Wrench Plane-Based Feature Set: Features are extracted using the 30 force/torque-related metrics computed using plane-based decomposition approach (i.e., 15 forcerelated metrics and 15 torque-related metrics).

Accordingly, two classifiers were trained with data coming from 8 trials considering all the subjects' data. After training, we tested the classifiers with the remaining data of 4 trials. The results indicate that WSB classifier achieves an accuracy of $78.59 \%$ and BER of 0.20 , whereas the WPB classifier achieves an accuracy of $91.02 \%$ and BER of 0.08 . The confusion matrices for the both classifiers are shown in Figure 4.

The results show that although both classifiers can successfully separate all behaviors, the performance is better with the WPB feature set. This means that the features extracted by the plane-based decomposition are more informative than those extracted by the space-based technique.

As mentioned in Section V-B, L1O cross-validation was used to investigate how the best achieving feature set performs

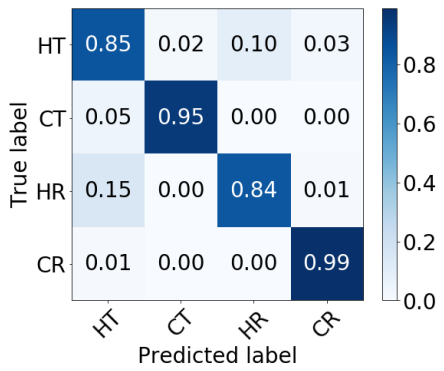

Fig. 5: Confusion matrix for classifier trained with WPB feature set utilizing L1O protocol. HT, CT, HR, and CR denote harmonious translation, conflicting translation, harmonious rotation, and conflicting rotation, respectively.

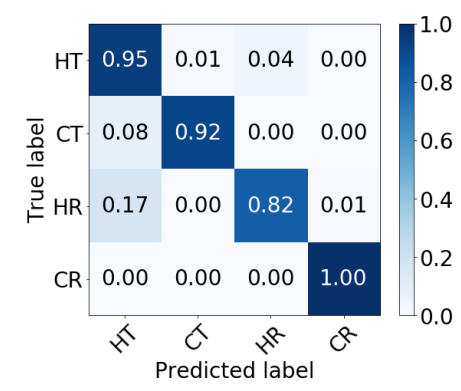

Fig. 6: Confusion matrix for classifier trained with the Optimal WPB feature set containing 25 features.

when the data comes from a new subject. We tested this procedure with the WPB feature set, and achieved an average accuracy of $88.69 \%$ and average BER of 0.094 . Figure 5 shows the average values for the normalised confusion matrices for this classifier across all users. As can be seen, the classification rates are consistent with the original protocol, and demonstrates good generalisation for different users.

\section{B. The Optimal Feature Set Selection}

To conclude our analyses, we performed feature selection to discover the optimal feature set to train the classification model. We used the mean decrease impurity (MDI) method for feature ranking [2] on the best achieving feature set, WPB. MDI represents importance regarding how many times a feature is used to split a node, weighted by the number of samples in the node. The decrease in node impurity is summed for each feature and averaged across all trees in the forest. In order to select the most relevant subset of features, we perform forward selection: Starting with an empty subset, we iteratively add the features to the model, based on the feature importance as given by MDI. Then, for each feature subset, we compute the performance of the model.

The optimal feature set that maximizes classification accuracy consists of 25 features. a detailed discussion of the optimal features can be found in Appendix C. Figure 6 shows the confusion matrix for the classifier, which achieves a classification accuracy of $91.25 \%$ and a BER of 0.07 .

\section{CONCLUSIONS}

In this study, we tested our hypothesis that haptics-related features would be sufficient to distinguish between interactive behavior patterns. We designed and conducted a pHHI 
experiment with 12 subjects, and investigated conflict-driven interaction behaviors under six task scenarios.

We designed a wrench-based feature set, inspired by the work of Noohi and Žefran [1], to classify the interaction behaviors. Using a random forest classifier, we verified that haptic information, i.e., wrenches, was sufficient to differentiate between the interactions patterns. This contrasts with our earlier work [25], [26], which suggested that kinematic information is needed to improve classification rates in distinguishing between interaction patterns in dyadic co-manipulation. In this work, we have demonstrated that using carefully devised haptic-related metrics derived from raw data eliminates the dependency on kinematics. This result is very important in designing future studies as it removes the necessity for tracking object motion when trying to understand the interaction states.

Another contribution of this work is our task analysis in different movement planes. By decomposing the motion in the object frame, we could eliminate the dominant forces for lifting motion, which would otherwise be interfering with the classification results. The proposed force decomposition method allowed more informative features to be extracted from raw data.

This study focuses on designing interaction-specific haptic features to identify conflict-related pHHI behaviors. Our results provide important insight into the amount of information that is carried over haptics in physical co-manipulation. Although this paper only presents an evaluation of pHHI behaviors, the outcomes of this work will be useful to design proactive collaborative robots, which are aware of the interaction states during the tasks.

In future work, we will use the proposed features with online classification as proposed in [26], and develop a system that identify the current interaction state in real-time during collaboration. Although this experiment does not cover all the human-human interaction patterns, this information will be useful when programming a robot partner that reads interaction states and reactively acts in response to the predicted behaviors, which is the ultimate goal of this research.

In this experiment, we constrained the subjects to a single scenario and asked them to concede to the experimenter's motion if they feel a conflict. This was done to avoid implicit role allocations between partners, and ease the data annotation process in our experiment. However, role definitions are key in human interactions and were demonstrated to be effective mechanisms to improve collaboration in our earlier work [5], [6], [15]. In future work, we plan to explore online interaction behavior classification as a way to investigate how conflicts arise and are resolved if subjects are also allowed to take on leadership roles.

\section{APPENDIX A}

\section{Force/TORQUE PATTERNS IN INTERACTION BEHAVIORS}

This section presents observations on the force/torque patterns encountered in the designed scenarios. These have guided the metric definitions and the feature extraction process, which are explained in Sections IV and V.
Figure 7 displays the forces and torques generated by a representative dyad in scenarios $\mathrm{Sc} 4$ and Sc5. In all plots, subscripts $S$ and $E$ refer to the subject and experimenter, respectively. Please note that all data are reported with respect to the object frame.

Figure 7(a) illustrates the forces, $\boldsymbol{f}$, exerted by each partner. Figures 7(b) and 7(c) respectively plot the computed torques, $\boldsymbol{\tau}^{c}$, and the measured torques, $\boldsymbol{\tau}^{m}$. The torques applied by each partner (i.e., $\boldsymbol{\tau}=\boldsymbol{\tau}^{c}+\boldsymbol{\tau}^{m}$ ) are depicted in Figure $7(\mathrm{~d})$. Finally, Figure 7(e) shows the net torques, $\tau_{\text {sum }}=\tau_{E}+\tau_{S}$, applied on the object by the dyad.

As shown in Figure 7(a), the forces exerted in $x$-axis have high values and show a similar pattern throughout all four behaviors. This is due to the fact that these forces are used dominantly for gravity compensation. Likewise, the partners exert matching torques about $y$-axis in opposite directions $\left(\tau_{\text {sum }_{y}} \approx 0\right)$ with a similar pattern throughout all behaviors when lifting the object (see Figures 7(d) and 7(e)).

During HT behavior (marked pink in Figure 7), the partners harmoniously translate the object along a desired axis. For instance, in scenario Sc4 (see Figure 2(a.IV)), the partners harmoniously translate the object along the positive $y$-axis as they share an intention to translate the object to the red target without rotation. This behavior manifests itself by the partners' applied forces along the direction of motion. In Figure 7(a) left panel, both partners apply forces in $y$-axis to synchronously accelerate the object, and then reverse the direction of the applied forces to decelerate it. Other examples of HT can be seen in scenario Sc5 (see Figure 2(a.V)).

In CT behavior (marked yellow in Figure 7), the partners disagree on the intended translation motion. This behavior is generated in scenario Sc4, where the experimenter's goal is to terminate the task at the red dot as shown in Figure 2(a.IV), whereas the subject's intended target is the black dot. This disparity between the goals creates a conflict, which is observable through investigating the forces along the $z$-axis. As shown in Figure 7(a) left panel, the subject wants to translate the object after reaching the red dot; hence he/she applies a force along the $z$-direction. However, the experimenter aims to stay on the red dot; thus, conflicting with the subject's translation intention by applying a force in the opposing direction.

In CR behavior (marked grey in Figure 7), the partners disagree on the intended rotation of the object. In this scenario, the subject aims to rotate the object at the beginning of the task. However, as shown in Figure 2(a.IV), the experimenter's scenario Sc4 does not involve that initial rotation. Therefore, the experimenter conflicts the subject and tries to prevent $\mathrm{him} / \mathrm{her}$ from rotating the object. In effect, both partners apply forces along the $y$-axis; however, since their intentions are different, these forces are expected to be in the same direction to generate opposing torques, which should cancel out as long as the conflict is not resolved. However, investigating Figures 7(a) and 7(b) left panel, we observe that the exerted forces on partners' sides along $y$-axis are in opposite directions, creating torques in the same direction (i.e., the computed torques $\tau^{c}$ around $x$-axis). Nevertheless, inspecting the object pose reveals that the experimenter succeed in preventing the object rotation. This can be clarified by observing the torques 
measured by the sensors on the handles, $\boldsymbol{\tau}^{m}$, which indicate the dominant opposition in torques about $x$-axis (see Figure 7(c) left panel). Summing up $\boldsymbol{\tau}^{c}$ and $\boldsymbol{\tau}^{m}$ to compute the applied torques on each partner's handle, $\tau$, reveals that opposing torques of comparable magnitudes are exerted on the partners' handles about $x$-axis. This results in approximately zero net torque on the object about the $x$-axis, $\tau_{\text {sum }_{x}}$, as shown in Figure 7(e) left panel, causing no apparent rotation in the
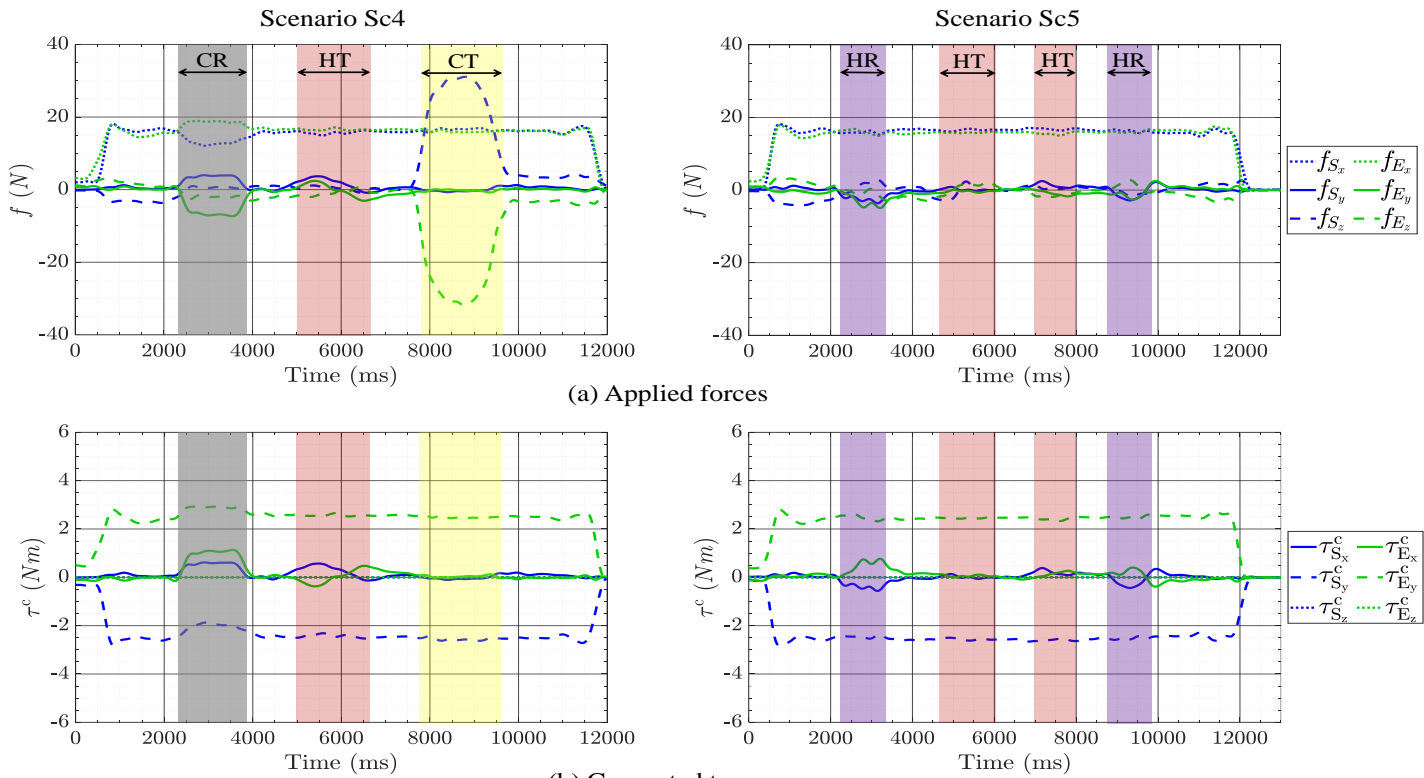

(b) Computed torques
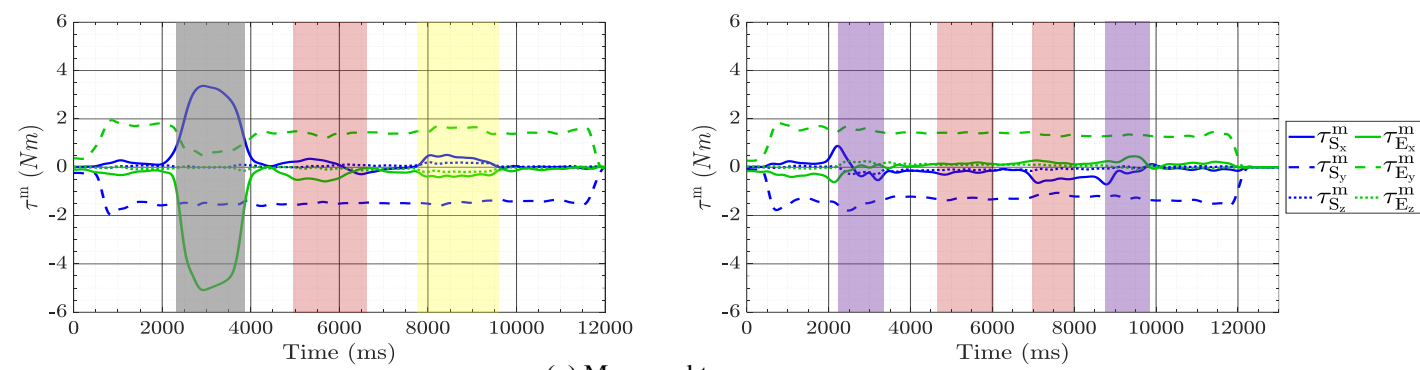

(c) Measured torques
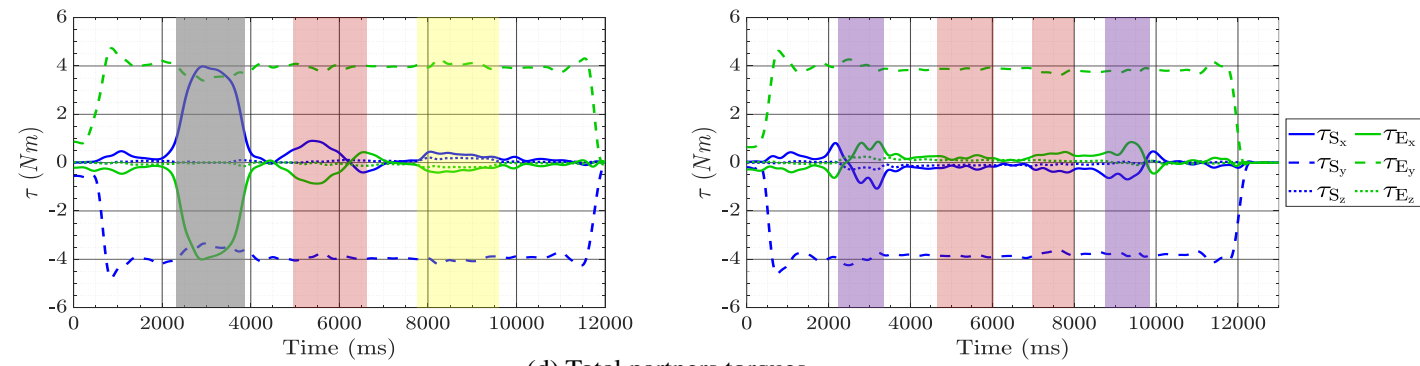

(d) Total partners torques
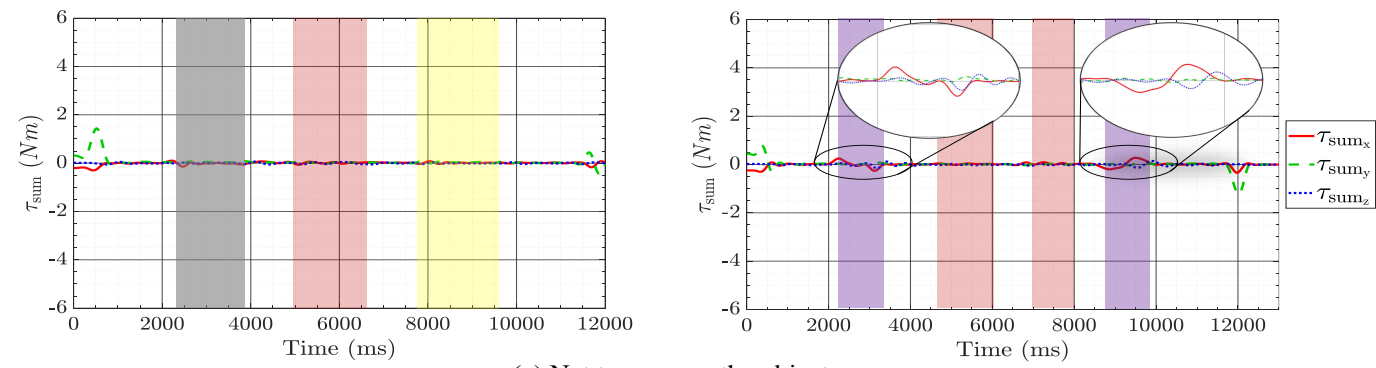

(e) Net torques on the object

Fig. 7: Forces and torques generated by a representative dyad under Sc4 and Sc5 for different interaction behaviors. Left and right panels represent the behaviors encountered during Sc4 and Sc5, respectively. CR, HT, CT, and HR refer to conflicting rotation (gray color), harmonious translation (pink color), conflicting translation (yellow color), and harmonious rotation (violet color) periods, respectively. Subscripts $S$ and $E$ refer to the subject and experimenter, respectively. (a) measured (applied) forces $\boldsymbol{f}$; (b) computed torques $\boldsymbol{\tau}^{c}$; (c) measured torques $\tau^{m}$; (d) total applied torques by each partner $\boldsymbol{\tau}$; (e) net torques applied on the object by the dyad, $\boldsymbol{\tau}_{s u m}=\boldsymbol{\tau}_{E}+\boldsymbol{\tau}_{S}$. 
final motion.

Finally, in HR behavior (marked violet in Figure 7), the partners share the intention to rotate the object in the same direction. Consequently, they work cooperatively by applying torques in the same direction about the axis of rotation. However, we see that the torques applied by partners about $x$-axis are in opposite directions as illustrated in Figures 7(b)(d) right panel. An explanation for this ambiguity is the out of synchronization of partners since each of them has his/her own reaction time to start the movement. In our experiments, we observed that it is hard for the dyad to synchronize their movement when rotating the object. In reality, the rotation is initiated by one partner, who applies a torque in the direction of rotation. This torque, due to the difference in partners' reaction time [20], produces a reaction torque on the second partner's handle. Since the second partner shares the same intention to rotate the object cooperatively, he/she tries to contribute to the rotation by compensating the reaction torque and adjusts the torque to provide the relevant compliance to his/her partner. This observation is consistent with Stefanov et al.'s observation in [19] about executor and conductor roles in dyadic operation. Figure 7(d) right panel illustrates $\tau_{S}$ and $\tau_{E}$. The torque exerted by one partner about $x$-axis is higher and in the opposite direction than the torque exerted by the second partner. As a result, some net torque is applied on the object by the dyad about the $x$-axis $\left(i . e ., \tau_{\text {sum }_{x}}\right)$, which generates the resulting rotation of the object (see Figure 7(e) right panel).

The conclusions we reach from these observations are as follows:

1) Each interaction behavior generates unique and complex force or torque patterns. Due to the complexity of the signals, a machine learning approach is needed to differentiate the interaction behaviors.

2) Using forces or torques in isolation would be inadequate to classify the behaviors. Hence, the integration of force and torque information is essential to describe the motion patterns in each of the four interaction behaviors.

3) Due to the nature of the manipulation task, some force and torque variables (e.g., those used for gravity compensation) may have significantly higher values, without being informative for classification. Hence, a planebased analysis is desirable.

\section{APPENDIX B}

\section{Classification With IsOlated ForCE AND TORque-BASED FEATURE SETS}

In Appendix A, we observed that while the force information can sufficiently describe the motion in some behaviors (e.g., HT), torque information is more informative in describing the motion in other behaviors (e.g., HR). Accordingly, we argued that relying on neither the force nor torque features alone would be sufficient.

In order to demonstrate our argument, we trained 4 classification models with 4 different feature sets (see Table III):

FSB Force Space-Based Feature Set: Features are extracted using the 5 force-related metrics computed using spacebased decomposition approach.

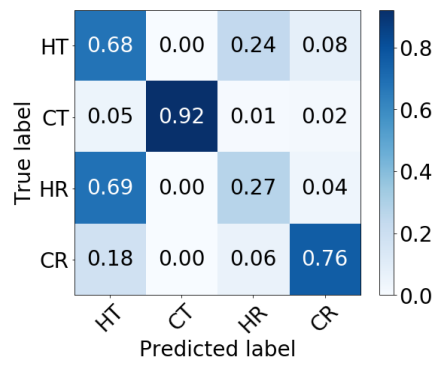

(a)

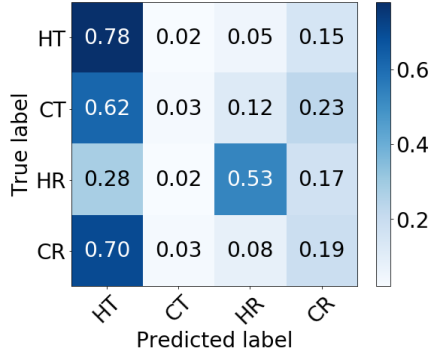

(c)

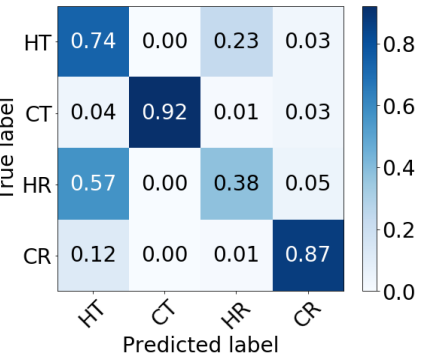

(b)

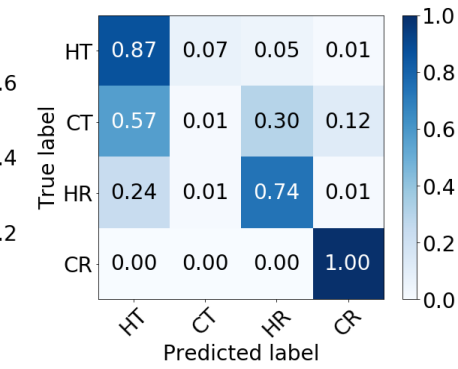

(d)
Fig. 8: Confusion matrices for classifiers trained with a) FSB: Force Space-Based b) FPB: Force Plane-Based c) TSB: Torque

Space-Based and d) TPB: Torque Plane-Based feature sets.

FPB Force Plane-Based Feature Set: Features are extracted using the 15 force-related metrics computed using planebased decomposition approach.

TSB Torque Space-Based Feature Set: Features are extracted using the 5 torque-related metrics computed using spacebased decomposition approach.

TPB Torque Plane-Based Feature Set: Features are extracted using the 15 torque-related metrics computed using plane-based decomposition approach.

The classifiers trained with FSB and FPB respectively achieve classification accuracies of $60.68 \%$ and $68.06 \%$ with BERs of 0.34 and 0.27 . Figures $8(a)$ and $8(b)$ show the confusion matrices for each feature set. As seen here, all classifiers are unsuccessful in distinguishing between HR and HT patterns, and score as poor as random guessing (i.e., $25 \%$ ) when trained with FSB. Using the plane-based features improves classification rates slightly, however does not manage to solve the confusion between HR and HT.

The classifiers trained with TSB and TPB achieve classification accuracies of $47.92 \%$ and $71.37 \%$ and BERs of 0.61 and 0.34 , respectively. The confusion matrices are shown in Figures 8(c) and 8(d). Evidently, the torque-based features also perform very poorly. Using the space-based decomposition, the classifier fails to distinguish CT, CR and HR. Adopting the plane based approach drastically improves the classification of CR. However, neither feature set (i.e., TSB and TPB) can improve the classification rate of CT.

These results show that the plane-based features lead to higher accuracies in comparison to those trained with features extracted using the space-based approach. However, these features cannot eliminate the confusion between HT vs HR using only force-related features and have failed in classifying CT correctly with the torque-related feature sets. 


\section{APPENDIX C \\ The Optimal Feature Selection}

As mentioned in Section VI-B, the final step in our analyses was the selection of an optimal feature set to train the classification model. Table IV demonstrates the feature ranking provided by MDI.

We have discussed that the optimal feature set consists of the first 25 features in this table, achieving a classification accuracy of $91.25 \%$ and a BER of 0.07 . Figure 9 shows that the accuracy is almost constant after including the first 13 features (89.78 accuracy, 0.09 BER), and reaches a good level with only 5 features (85.66 accuracy, 0.12 BER). We observe a drop in classification accuracy when the feature subset is limited to the first 4 features (69.62 accuracy, 0.35 BER). Figure 10 shows the confusion matrices for the classifiers trained with feature subsets consisting of 13,5 and 4 features.

\section{TABLE IV}

FEATURE RANKING PROVIDED By MDI. FOR EACH METRIC, SUPERSCRIPTS $\tau$ AND $f$ STAND FOR TORQUE AND

FORCE. SUBSCRIPTS $E, S, e, t e, n e$, AND $s$ STAND FOR

EXPERIMENTER, SUBJECT, INDIVIDUAL, TEAM, NEGOTIATION, AND SIMILARITY, RESPECTIVELY.

\begin{tabular}{|c|c|c||c|c|c||c|c|c|}
\hline Rank & Metric & $\begin{array}{c}\text { Importance } \\
(\%)\end{array}$ & Rank & Metric & $\begin{array}{c}\text { Importance } \\
(\%)\end{array}$ & Rank & Metric & $\begin{array}{c}\text { Importance } \\
(\%)\end{array}$ \\
\hline $\mathbf{1}$ & $M_{e_{S}}^{\tau}(y)$ & 12.39 & $\mathbf{1 1}$ & $M_{n e}^{\tau}(x)$ & 3.92 & $\mathbf{2 1}$ & $M_{t e}^{f}(y z)$ & 1.32 \\
\hline $\mathbf{2}$ & $M_{e_{S}}^{\tau}(x)$ & 10.90 & $\mathbf{1 2}$ & $M_{t_{e}}^{f}(x z)$ & 3.19 & $\mathbf{2 2}$ & $M_{n e}^{\tau}(z)$ & 0.63 \\
\hline $\mathbf{3}$ & $M_{e_{E}}^{\tau}(y)$ & 9.80 & $\mathbf{1 3}$ & $M_{e_{E}}^{\tau}(z)$ & 2.94 & $\mathbf{2 3}$ & $M_{s}^{f}(y z)$ & 0.54 \\
\hline $\mathbf{4}$ & $M_{t_{e}}^{\tau}(x)$ & 8.72 & $\mathbf{1 4}$ & $M_{e_{S}}^{\tau}(z)$ & 2.58 & $\mathbf{2 4}$ & $M_{n e}^{\tau}(y)$ & 0.41 \\
\hline $\mathbf{5}$ & $M_{e_{S}}^{f}(x y)$ & 7.47 & $\mathbf{1 5}$ & $M_{t e}^{\tau}(z)$ & 2.31 & $\mathbf{2 5}$ & $M_{t e}^{\tau}(y)$ & 0.39 \\
\hline $\mathbf{6}$ & $M_{e_{E}}^{\tau}(x)$ & 6.78 & $\mathbf{1 6}$ & $M_{n e}^{f}(y z)$ & 2.05 & $\mathbf{2 6}$ & $M_{s}^{\tau}(x)$ & 0.34 \\
\hline $\mathbf{7}$ & $M_{e_{E}}^{f}(x z)$ & 4.17 & $\mathbf{1 7}$ & $M_{e_{E}}^{f}(y z)$ & 1.89 & $\mathbf{2 7}$ & $M_{s}^{\tau}(z)$ & 0.11 \\
\hline $\mathbf{8}$ & $M_{e_{S}}^{f}(x z)$ & 4.08 & $\mathbf{1 8}$ & $M_{e_{S}}^{f}(y z)$ & 1.71 & $\mathbf{2 8}$ & $M_{n e}^{f}(x y)$ & 0 \\
\hline $\mathbf{9}$ & $M_{t_{e}}^{f}(x y)$ & 4.05 & $\mathbf{1 9}$ & $M_{s}^{f}(x y)$ & 1.63 & $\mathbf{2 9}$ & $M_{n e}^{f}(x z)$ & 0 \\
\hline $\mathbf{1 0}$ & $M_{e_{E}}^{f}(x y)$ & 4.04 & $\mathbf{2 0}$ & $M_{s}^{f}(x z)$ & 1.50 & $\mathbf{3 0}$ & $M_{s}^{\tau}(y)$ & 0 \\
\hline
\end{tabular}

Although the classifier trained with an optimal feature set consisting of 25 features achieves the higher accuracy with a minimum classification rate of $82 \%$ for $\mathrm{HR}$, the classifiers trained with 13 and 5 features also achieve good accuracies with a minimum classification rate of $70 \%$ for HR. On the other hand, when we train the classifier with only the first 4 features, the overall accuracy drops to $69 \%$ with strong misclassification for the CT behavior. Observing Table IV reveals that this feature set does not contain any force information. This indicates the necessity of force information to describe the translation behaviors (i.e., HT and CT) as we have observed in Appendix A.

Moreover, in Table IV, we can see that the first ten most important ranks were occupied equally by both force- and torque-related metrics which indicates the importance of using a combination of force and torque information in describing the interaction between the partners. This coincides with our observation in Appendix A about how the force and torque data are complement each other in describing the interaction behaviors during the manipulation process.

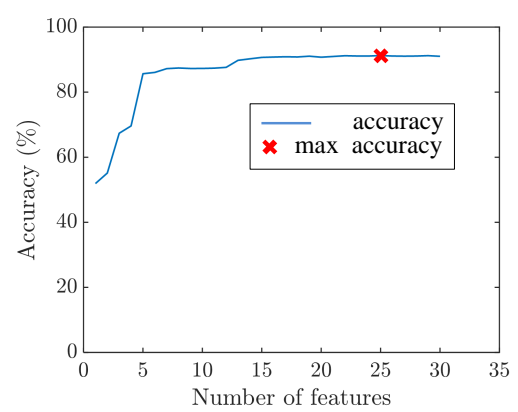

Fig. 9: Classification accuracy vs number of features added to the model incrementally using MDI ranking.

Furthermore, our haptic metrics can be categorized into two groups; those that include information about the forces/torques in the considered plane only (i.e., negotiation efficiency and similarity); and those that carry the force/torque information in the considered plane with respect to total forces/torques applied by the partners (i.e., individual efficiency and team efficiency). Looking at Table IV, we can conclude that the metrics in the latter category are in general more significant than those in the first category. For instance, it is shown that negotiation force efficiency in $x-y$ plane $\left(28^{\text {th }}\right)$, and in $x-z$ plane $\left(29^{\text {th }}\right)$ and the similarity in torques about $y$-axis have zero weight, as these features hold no distinct information throughout all interaction patterns.

\section{ACKNOWLEDGMENT}

Z.A. acknowledges the financial support by University of Baghdad. C.B. acknowledges the financial support by TUBITAK under the contract number of 117E645. A.K. acknowledges the financial support by EPSRC ("HEAP", EP/S033718/1; "NCNR”, EP/R02572X/1; “CoRSA”, 742782).

\section{REFERENCES}

[1] E. Noohi and M. Žefran, "Quantitative measures of cooperation for a dyadic physical interaction task," in IEEE-RAS International Conference on Humanoid Robots, 2014, pp. 469-474.

[2] L. Breiman, "Random forests," Machine Learning, vol. 45, no. 1, pp. 5-32, 2001.

[3] C. Basdogan, C. HO, M. A. Srinivasan, and M. Slater, "An experimental study on the role of touch in shared virtual environments," $A C M$ Transactions on Computer-Human Interaction (TOCHI), vol. 7, no. 4, pp. 443-460, 2000.

[4] J. Moll and E.-L. Sallnäs, "Communicative functions of haptic feedback," in International Conference on Haptic and Audio Interaction Design. Springer, 2009, pp. 1-10.

[5] A. Kucukyilmaz, S. O. Oguz, T. M. Sezgin, and C. Basdogan, "Improving human-computer cooperation through haptic role exchange and negotiation," in Immersive Multimodal Interactive Presence. Springer, 2012, pp. 229-254.

[6] A. Kucukyilmaz, T. M. Sezgin, and C. Basdogan, "Intention recognition for dynamic role exchange in haptic collaboration," IEEE Transactions on Haptics, vol. 6, no. 1, pp. 58-68, 2013.

[7] R. Groten, D. Feth, R. L. Klatzky, and A. Peer, "The role of haptic feedback for the integration of intentions in shared task execution," IEEE Transactions on Haptics, vol. 6, no. 1, pp. 94-105, 2013.

[8] G. Ganesh, A. Takagi, R. Osu, T. Yoshioka, M. Kawato, and E. Burdet, "Two is better than one: Physical interactions improve motor performance in humans," Scientific Reports, vol. 4, 2014.

[9] A. Takagi, F. Usai, G. Ganesh, V. Sanguineti, and E. Burdet, "Haptic communication between humans is tuned by the hard or soft mechanics of interaction," PLoS Computational Biology, vol. 14, no. 3, pp. 1-17, 2018. 


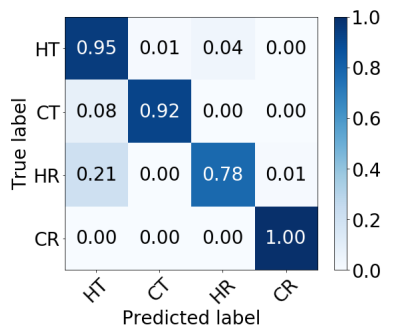

(a)

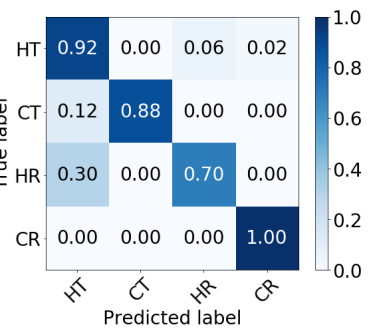

(b)

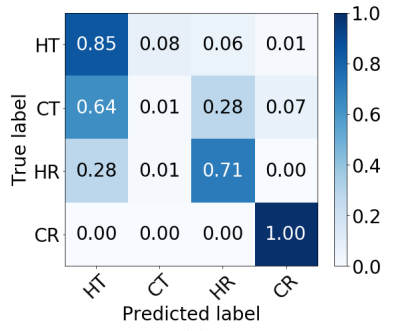

(c)

Fig. 10: Confusion matrices for classifier trained with (a) 13 features, (b) 5 features, and (c) 4 features from the optimal WPB feature set.

[10] A. Takagi, M. Hirashima, D. Nozaki, and E. Burdet, "Individuals physically interacting in a group rapidly coordinate their movement by estimating the collective goal," Neuroscience, no. 8, pp. 1-19, 2019.

[11] L. Marchal-Crespo, J. Furumasu, and D. Reinkensmeyer, "A robotic wheelchair trainer: design overview and a feasibility study," Journal of NeuroEngineering and Rehabilitation, vol. 7, no. 1, pp. 1-12, 2010. [Online]. Available: http://dx.doi.org/10.1186/1743-0003-7-40

[12] S. Parastegari, E. Noohi, B. Abbasi, and M. Žefran, "Failure recovery in robot-human object handover," IEEE Transactions on Robotics, vol. 34, no. 3, pp. 660-673, 2018.

[13] E. A. Mielke, E. C. Townsend, and M. D. Killpack, "Analysis of rigid extended object co-manipulation by human dyads: Lateral movement characterization," arXiv preprint arXiv:1702.00733, 2017.

[14] E. Mielke, E. Townsend, D. Wingate, and M. D. Killpack, "Humanrobot co-manipulation of extended objects: Data-driven models and control from analysis of human-human dyads," arXiv preprint arXiv:2001.00991, 2020.

[15] A. Mörtl, M. Lawitzky, A. Kucukyilmaz, M. Sezgin, C. Basdogan, and S. Hirche, "The role of roles: Physical cooperation between humans and robots," The International Journal of Robotics Research, vol. 31, no. 13, pp. 1656-1674, 2012.

[16] S. O. Oguz, A. Kucukyilmaz, T. M. Sezgin, and C. Basdogan, "Supporting negotiation behavior with haptics-enabled human-computer interfaces," IEEE Transactions on Haptics, vol. 5, no. 3, pp. 274-284, 2012.

[17] N. Jarrassé, V. Sanguineti, and E. Burdet, "Slaves no longer: review on role assignment for human-robot joint motor action," Adaptive Behavior, vol. 22, no. 1, pp. 70-82, 2014.

[18] K. B. Reed and M. A. Peshkin, "Physical collaboration of human-human and human-robot teams," IEEE Transactions on Haptics, vol. 1, no. 2 , pp. 108-120, 2008.

[19] N. Stefanov, A. Peer, and M. Buss, "Role determination in human-human interaction," in Third Joint EuroHaptics Conference and Symposium on Haptic Interfaces for Virtual Environment and Teleoperator Systems. IEEE, 2009, pp. 51-56.

[20] A. Takagi, N. Beckers, and E. Burdet, "Motion plan changes predictably in dyadic reaching," PLoS one, vol. 11, no. 12, p. e0167314, 2016.

[21] A. Melendez-Calderon, V. Komisar, G. Ganesh, and E. Burdet, "Classification of strategies for disturbance attenuation in human-human collaborative tasks," in Annual International Conference of the IEEE Engineering in Medicine and Biology Society, 2011, pp. 2364-2367.

[22] N. Jarrassé, T. Charalambous, and E. Burdet, "A framework to describe, analyze and generate interactive motor behaviors," PLoS $O N E$, vol. 7, no. 11, p. e49945, 2012. [Online]. Available: http://dx.doi.org/10.1371/journal.pone.0049945

[23] Y. Li, G. Carboni, F. Gonzalez, D. Campolo, and E. Burdet, "Differential game theory for versatile physical human-robot interaction," Nature Machine Intelligence, vol. 1, no. 1, pp. 36-43, 2019.

[24] D. J. Agravante, A. Cherubini, A. Sherikov, P.-B. Wieber, and A. Kheddar, "Human-humanoid collaborative carrying," IEEE Transactions on Robotics, vol. 35, no. 4, pp. 833-846, 2019.

[25] C. E. Madan, A. Kucukyilmaz, T. M. Sezgin, and C. Basdogan, "Recognition of haptic interaction patterns in dyadic joint object manipulation," IEEE Transactions on Haptics, vol. 8, no. 1, pp. 54-66, 2015.

[26] A. Kucukyilmaz and I. Issak, "Online identification of interaction behaviors from haptic data during collaborative object transfer," IEEE Robotics and Automation Letters, vol. 5, no. 1, pp. 96-102, 2020.

[27] S. Garrido-Jurado, R. Muñoz-Salinas, F. J. Madrid-Cuevas, and M. J. Marín-Jiménez, "Automatic generation and detection of highly reliable fiducial markers under occlusion," Pattern Recognition, vol. 47, no. 6, pp. 2280-2292, 2014.

[28] F. Pedregosa, G. Varoquaux, A. Gramfort, V. Michel, B. Thirion, O. Grisel, M. Blondel, P. Prettenhofer, R. Weiss, V. Dubourg, J. Vander- plas, A. Passos, D. Cournapeau, M. Brucher, M. Perrot, and E. Duchesnay, "Scikit-learn: Machine learning in Python," Journal of Machine Learning Research, vol. 12, pp. 2825-2830, 2011.

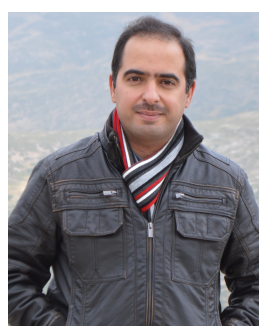

Zaid Al-Saadi is a Ph.D. candidate in mechanical engineering department at Koç University, Istanbul. $\mathrm{He}$ received his B.Sc. and M.Sc. degrees in Mechanical Engineering from Mustansiriyah University, Baghdad, in 2002 and 2007, respectively. His research interests include haptics, physical human-X interaction, and robotics.

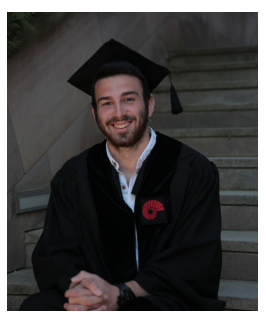

Doganay Sirintuna received his B.Sc. and M.Sc. degrees in Mechanical Engineering from Koç University, Istanbul, in 2018 and 2020, respectively. He was granted the Academic Excellence Award at his graduation from Koç University in 2020. His research interests include physical human-robot interaction, robotics, mechatronics, and robot learning.

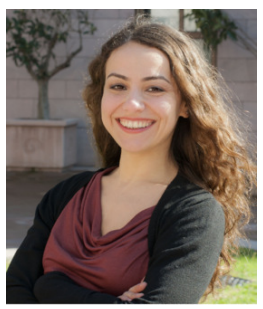

Ayse Kucukyilmaz (S'11, M'14) is an assistant professor in Computer Science at University of Nottingham, UK, and a member of the Lincoln Centre for Autonomous Systems (L-CAS) in the University of Lincoln, UK. Her research interests include haptics, physical HRI, assistive robotics, and machine learning. Previously, Dr Kucukyilmaz worked as a postdoctoral research associate at the Personal Robotics Laboratory of Imperial College London, and was a visiting researcher at the InformationOriented Control group in Technical University of Munich in 2011. She received her Ph.D. in Computational Sciences and Engineering from Koç University, Turkey in 2013. She was the recipient of the Academic Excellence Award at her graduation from Koç University in 2013 and the 2016 Meritorious Service Award for her work as a reviewer for the IEEE Transactions on Haptics. She has received individual fellowships from Marie Sklodowska-Curie Actions and TUBITAK, and holds grants from UKRI EPSRC and European Commission.

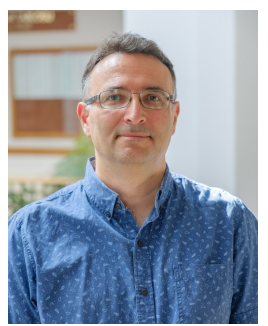

Cagatay Basdogan received the Ph.D. degree in Mechanical Engineering from Southern Methodist University, in 1994. He is a faculty member in the mechanical engineering and computational sciences and engineering programs at Koç University, Istanbul, Turkey. He is also the director of the Robotics and Mechatronics Laboratory, Koç University. Before joining Koç University, he worked at NASA JPL/Caltech, MIT, and Northwestern University Research Park. His research interests include haptics, robotics, mechatronics, biomechanics, medical simulation, computer graphics, and multi-modal virtual environments. He served as the associate editor in chief of the IEEE Transactions on Haptics from 2015-2020. In addition to serving in program and organizational committees of several haptics conferences, and editorial board of journals, he chaired the IEEE World Haptics Conference in 2011. 